\title{
Overexpression of PIK3R1 promotes hepatocellular carcinoma progression
}

\author{
Xuejun Ai ${ }^{1,2+}$, Lei Xiang ${ }^{3+}$, Zhi Huang ${ }^{1,3}$, Shi Zhou ${ }^{3}$, Shuai Zhang ${ }^{3}$, Tao Zhang ${ }^{1,2^{*}}$ and Tianpeng Jiang , $^{1,3^{*}}$
}

\begin{abstract}
Background: Phosphoinositide-3-kinase, regulatory subunit 1 (PIK3R1) could regulate cancer cell proliferation important for cancer cell proliferation; however, its role in Hepatocellular carcinoma (HCC) remains largely unknown. Here, we investigated the role of PIK3R1 in HCC and examined the underlying molecular mechanisms.

Methods: The expression of PIK3R1 was evaluated by immunohistochemistry and ART-PCR in a series of HCC tissues. The mRNA and protein expression of PIK3R1 was used by qRT-PCR and western blot assays in a series of human HCC cell lines, and then we choose MHCC97H and HCCLM3 cells as a model to investigate the effect of PIK3R1 on HCC progression. The effects of PIK3R1 knowdown on cell proliferation, migration, apoptosis of HCC were assessed by the MTT assay, clonogenic assays, wound healing assay and flow cytometry in vitro. Western blot assay was performed to assess the expression changes of PI3K/AKT/mTOR signaling pathway.
\end{abstract}

Results: Our results found that PIK3R1 was highly expressed in HCC tissues compared with adjacent normal tissues. Knockdown of PIK3R1 inhibited the proliferation, migration and promoted apoptosis of HCC cell lines. In addition, we proved that knockdown of PIK3R1 downregulated p-PI3K, p-AKT, and p-mTOR expressions in MHCC97H and HCCLM3 cells.

Conclusions: In conclusion, PIK3R1 providing potential novel targets for the treatment of HCC.

Keywords: Hepatocellular carcinoma, PIK3R1, Apoptosis, Targeted therapy

\section{Background}

Hepatocellular carcinoma (HCC) is the fifth most common cancer worldwide, and one of the leading cause of death among malignancies in China [1,2]. Despite advances in diagnostic and treatment modalities, the prognosis for HCC has not significantly improved, and the 5-year survival rate for patients with HCC remains poor, which is largely attributable to the high rates of distant metastasis [3]. Thus, there is an urgent need to develop new strategies to HCC treatment.

Phosphoinositide 3-kinase (PI3K) is a heterodimer that consists of an $\mathrm{SH} 2$-containing regulatory subunit (p85) and a catalytic subunit (p110), with both subunits expressed in multiple isoforms [4]. There are eight

\footnotetext{
*Correspondence: zhangtaodoctor@163.com; 26900988@qq.com

${ }^{\dagger}$ Xuejun Ai and Lei Xiang contributed equally to this work

${ }^{1}$ The Key Laboratory of Medical Molecular Biology, Guizhou Medical

University, Guiyang 550002, China

Full list of author information is available at the end of the article
}

isoforms of the regulatory subunit encoded by three different genes, Pik3R1, PIk3R2, and PIk3R3. In most eukaryotic cells, the gene products of PIk3R1 constitute $65-75 \%$ of the intracellular pool of regulatory subunits in the form of $\mathrm{p} 85 \alpha$ [5]. It has been reported that deletion of PIk3R1 in liver may result in a marked reduction in insulin-stimulated PI3K activity with significant defection in glucose and lipid homeostasis, as well as in hepatic size and function [6]. An increasing number of PIK3R1 have been identified to be differentially expressed in many human cancers and implicated in tumor progression and metastasis [7]. Aberrations of PIK3R1 occur in endometrial cancers (EC), breast cancer, colon cancer, and glioblastomas, as an important therapeutic target through inhibiting mTOR (http://www.sanger.ac.uk/ genetics/CGP/cosmic) [8]. However, the role of PIK3R1 in hepatocellular carcinogenesis remains unknown. In this study, we investigated the expression of PIK3R1 in human HCC by using qRT-PCR, western blot, and 
immunohistochemistry, and explored the potential role of PIK3R1 in HCC progression. In addition, we explored the possible mechanism PIK3R1 in human HCC.

\section{Methods}

\section{HCC tissue samples}

From 2005 to 2014, tumor samples and corresponding adjacent normal tissues were collected from 92 HCC patients receiving surgery at the Affiliated Baiyun Hospital of Guizhou Medical University. The corresponding adjacent normal tissue samples were obtained $>5 \mathrm{~cm}$ from the edge of the cancerous region and there were no obvious tumor cells evaluated by pathologist. These tissue samples were conserved in liquid nitrogen after collection or prepared in paraffin sections. No systemic or local treatment had been received before operation. Both tumor and nontumor tissues were histologically confirmed. All the tissue samples were obtained with informed consent from all the patients. This study was approved by the Institute Research Ethics Committee of Guizhou Medical University.

\section{Cell lines}

HCC cell lines MHCC97L, Huh7, HepG2, HCCLM3, SMMC-7721, MHCC97H and normal liver cell lines HL-7702 were from the tumor cell bank of Chinese Academy of Sciences. All the cell lines were grown in Dulbecco's modified eagle medium supplemented with $10 \%$ fetal bovine serum, $100 \mathrm{Ag} / \mathrm{AL}$ streptomycin, and $100 \mathrm{Ag} / \mathrm{AL}$ penicillin ( $\mathrm{pH}$ 7.2-7.4) in a humidified incubator containing $5 \% \mathrm{CO}_{2}$ at $37^{\circ} \mathrm{C}$.

\section{Immunohistochemistry}

For each patient sample, three paraffin sections of $5 \mu \mathrm{m}$ were prepared, for immunohistochemical staining. Sections were dewaxed using xylene, followed by hydration with ethanol solutions and addition of EDTA for antigen retrieval. Later, sections were blocked with normal goat serum for $30 \mathrm{~min}$ to eliminate non-specific binding. Sections were incubated with primary antibody against PIK3R1 (Abcam, Cambridge, UK). Sections were then incubated with biotin-labeled secondary antibodies for $30 \mathrm{~min}$ at room temperature, followed by staining with diaminobenzidine (DAB).

\section{Reverse transcription-quantitative PCR}

Total RNA of tissues or cultured cells was isolated by using TRIzol reagent (Invitrogen). Total RNA (1 $\mu \mathrm{g})$ was transcribed into cDNA by using a First-strand cDNA Synthesis System (Invitrogen). $1 \mu \mathrm{l}$ DNA template was used to amplify by using Power $\mathrm{SYBR}^{\circledR}$ Green PCR Master Mix (ABI, USA) on the 7500 real time PCR system (ABI, life technology). The reaction system was performed in a volume of $20 \mu \mathrm{l}$. The GAPDH was used as a loading control for each specific gene. Each experiment was performed three times and each sample was tested in triplicate. The sequences of human PIK3R1 primers

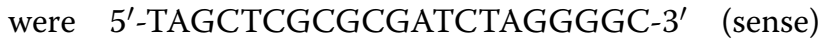
and $5^{\prime}$-CGCGATCAATAAAGCTAG-3' (antisense). The primers for human GAPDH were $5^{\prime}$-GCACCGTCAAGG CTGAGAAC- $3^{\prime}$ (sense) and 5'-TGGTGAAGACGCCAG TGGA-3' (antisense).

\section{Western blot analysis}

Whole cells were lysed on ice in a lysis buffer (RIPA, Beyotime, Shanghai, China) with a protease inhibitor mixture cocktail (Roche, Switzerland). After centrifugation at $12,000 \mathrm{rpm}$ for $30 \mathrm{~min}$ at $4{ }^{\circ} \mathrm{C}$, the protein concentrations of supernatants in samples were measured by the BCA protein assay (Thermo scientific, Rockford, IL, USA). Equal amounts of protein $(30 \mu \mathrm{g})$ were separated by $10-12 \%$ NUPAGE Bis-Tris Gel (Invitrogen, CA, USA) electrophoresis (constant voltage: $120 \mathrm{mv}$ ) and transferred onto polyvinylidene fluoride (PVDF, $0.45 \mu \mathrm{m}$ ) membranes (constant current: $350 \mathrm{~mA}$ for $70 / 120 \mathrm{~min}$ ). After being blocked by Tris-buffered saline and Tween 20 (TBST) buffer containing 5\% non-fat powder milk for $2 \mathrm{~h}$, the membranes were incubated with primary antibodies overnight on ice. After washing the membranes several times in TBST while agitating, detection was performed using the appropriate secondary HRP-conjugated antimouse or antirabbit antibody. Immunoreactive bands on the blots were visualized with enhanced chemiluminescence reagent ECL kit (Beit Haemek, Israel).

\section{Small interfering RNA transfection of human HCC cell line} Human PIK3R1 siRNA (5'-CCTAGCGCATATCGCC$\left.3^{\prime}\right)$ and control-siRNA were synthesized by GenePharma (shanghai, china). Cells were transfected with sh PIK3R1 or control-shRNA using Lipofectamine 2000 (Invitrogen, Life Technologies), according to the manufacturer's instructions.

\section{MTT assays}

The proliferation of cells was evaluated by the MTT assay. Cells were plated in a 96-well plate at $3 \times 10^{3}$ cells/ well and were allowed to grow for different times. The growth rate was determined by the cell number and was counted in triplicate every day by MTT assay. Briefly, cells were incubated with $50 \mu \mathrm{l}$ of $0.2 \%$ MTT for $4 \mathrm{~h}$ at $37{ }^{\circ} \mathrm{C}$ in a $5 \% \mathrm{CO}_{2}$ incubator. Following MTT incubation, $150 \mu \mathrm{l}$ of $100 \%$ DMSO was added to dissolve the crystals. Viable cells were counted every day by reading the absorbance at $490 \mathrm{~nm}$ using a 96-plate reader BP800 (Dynex Technologies). 


\section{Clone formation assay}

Clone formation assay was conducted to examine the effect of PIK3R1-siRNA on cell growth in HCC cell lines. $4 \times 10^{5}$ cells were plated in a 6 -well plate. After $24 \mathrm{~h}$ of transfection, the cells were trypsinized, and 1000 single viable cells were plated in three 6-well plates. The cells were then incubated for 14 days at $37{ }^{\circ} \mathrm{C}$ in the condition of $5 \% \mathrm{CO}_{2}$. Colonies were stained with $0.1 \%$ crystal violet, washed with water, and counted ten random fields manually. The colonies containing at least 100 cells were scored. The surviving fraction in PIK3R1-siRNA transfected cells was normalized to untreated control cells with respect to clonogenic efficiency.

\section{Wound healing assay}

Wound healing assay was adopted to test the migration ability of HCC cells. In our study, cells were digested after transfection by specific siRNA and control siRNA to human PIK3R1 for $24 \mathrm{~h}$ in 6-well plates, $2 \times 10^{5}$ cells were plated in 24-well plates, when cell confluence reached approximately $100 \%$, the old medium was removed and the monolayer was wounded by scratching with a $10-\mu \mathrm{l}$ sterile pipette tip lengthwise along the chamber, then cells were washed three times with PBS and cultured with serum-free medium at $37^{\circ} \mathrm{C}$. Images of migrating cells into the wound were photographed at $0 \mathrm{~h}$ and $48 \mathrm{~h}$ using an inverted microscope. The scratch width of the cells was confirmed by detecting the width of the monolayer wound at 0 and $48 \mathrm{~h}$, and the migration index was counted as follows: migration index $=(0 \mathrm{~h}$ scratch width $-48 \mathrm{~h}$ scratch width) $/ 0 \mathrm{~h}$ scratch width) $\times 100$ [9].

\section{Transwell migration assay}

Cell migration ability was determined by transwell assays. The treated MHCC97H and HCCLM3 cells $\left(1.0 \times 10^{5} /\right.$ $\mathrm{ml}$ ) were seeded in the upper chambers (BD Biosciences, NY, USA). The upper chamber was filled with serum-free medium and the lower chamber was supplemented with $10 \%$ fetal bovine serum. Hence this allowed the cells in the upper chamber migrate into the lower chamber. After $48 \mathrm{~h}$ incubation, the cells that had invaded through the membrane were stained with $0.1 \%$ crystal violet solution. The sections were observed by using a light microscope (magnification at $\times 100$ ).

\section{Apoptosis assay}

The apoptosis ability was measured by using Annexin V-FITC/PI apoptosis detection kit (BestBio, Shanghai, China). Cells were digested after transfection by specific siRNA and control siRNA to human PIK3R1 were washed with ice-cold PBS. The treated cells $\left(1 \times 10^{6}\right.$ cells $\left./ \mathrm{ml}\right)$ were suspended with $100 \mu \mathrm{l}$
$1 \times$ binding buffer and double stained with Annexin V-FITC/PI for 15 min according to the manufacturer's instructions. Intensities of fluorescence signals were measured on a FACS Calibur flow cytometer (BectonDickinson, Franklin-Lakes, NJ, USA). The image of apoptosis was divided into four quadrants: all living cells (double negative), early apoptotic cells (Annexin $\mathrm{V}$-positive, propidium iodide-negative), necrotic cells (Annexin V-negative, propidium iodide-negative positive), as well as late apoptotic cells (double positive). We counted the early apoptotic cells and the late apoptotic cells.

\section{Statistical analysis}

For continuous variables, data are expressed as mean \pm standard deviation (SD). The difference between PIK3R1 mRNA or protein expression in tumor tissue and that in adjacent normal tissues was evaluated using Student's t-test. GraphPad Prism 5.0 Software was employed to perform statistical analysis. All statistical tests were two-tailed and statistical significance was assumed for $\mathrm{p}<0.05$.

\section{Results}

PIK3R1 expression levels are significantly upregulated in human HCC tissue and HCC cell lines of high metastatic potential

qRT-PCR was performed to detect the expression of PIK3R1 mRNA in 92 paired HCC tissues and corresponding adjacent tissues. PIK3R1 expression was significantly high in HCC tissues compared with the related normal pericarcinomatous tissues (Fig. 1a). In addition, statistical results showed that PIK3R1 expression was closely associated with lymphatic metastasis $(\mathrm{p}=0.029)$, distal metastasis $(\mathrm{p}=0.004)$ and pathologic tumor, node, metastasis stage (TNM stage, $\mathrm{p}=0.002$ ), but not age $(\mathrm{p}=0.548)$, gender $(\mathrm{p}=0.484)$ and tumor size $(\mathrm{p}=0.503$, Table 1$)$. Immunohistochemical staining results showed that PIK3R1 expression in HCC specimens was significantly upregulated compare with adjacent non-tumoral liver tissue (Fig. 1b). Then, we detected the mRNA and protein expression of PIK3R1 in a series of human HCC cell lines, including MHCC97L, Huh7, HepG2, HCCLM3, SMMC-7721 and MHCC $97 \mathrm{H}$ by qRT-PCR and Western Blot analyses, respectively. Our results showed that HCCLM3 and MHCC $97 \mathrm{H}$ cells (high metastatic potential) showed the higher expression of PIK3R1 (Fig. 1c, d). Thus, we used MHCC97H and HCCLM3 cells as a model to investigate the effect of PIK3R1 on HCC progression. 


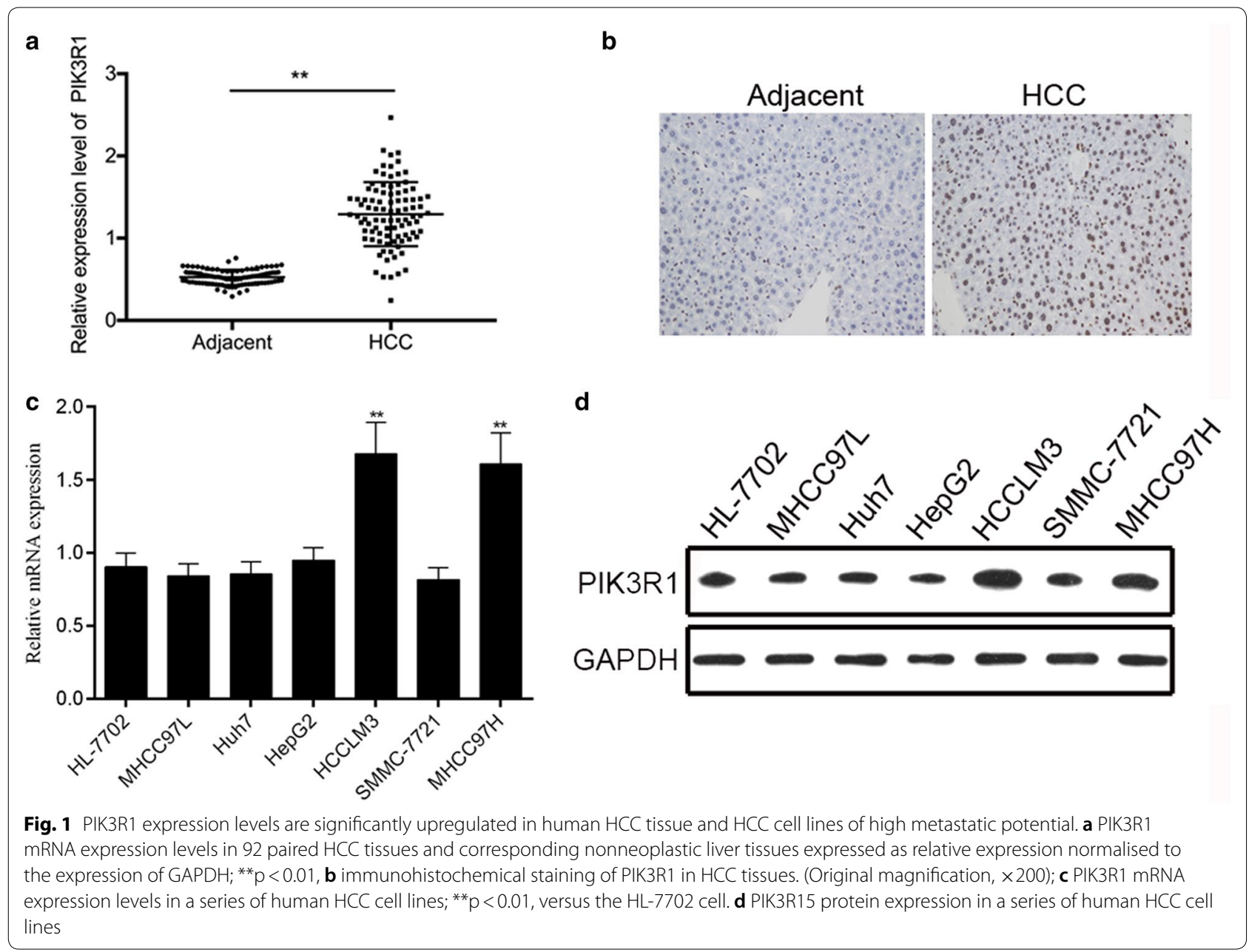

PIK3R1 associated directly with the ability of cell proliferation of HCC cell lines

To further assess the biological function of PIK3R1 in HCC, we used the siRNA to knockdown the PIK3R1 level. As shown in Fig. 2, PIK3R1 expression was distinctly decreased at mRNA and protein levels in MHCC97H and HCCLM3 compared with siRNA NC group, indicating that the specific siRNA of PIK3R1 effectively suppressed the expression of PIK3R1 in HCC cell lines.

To further test whether PIK3R1 were related to proliferation ability of HCC cells, we measured the effects of PIK3R1 expression levels on cancer cell proferation by MTT and Clonogenic assays. As shown in Fig. 3a, b, PIK3R1 knockdown was associated with significantly decreased cell viability of HCCLM3 and MHCC97H cells. Furthermore, PIK3R1 knockdown in HCCLM3 and MHCC97H cells consistently reduced the colony formation ability (Fig. 3c-f), suggesting that PIK3R1 may act as an oncogene involved in the promotion of $\mathrm{HCC}$ cell proliferation.
Next, we carried out scratch wound-healing and transwell assay to evaluated whether PIK3R1 regulated the ability of migration of HCC cells. We found that knowdown of PIK3R1 markedly diminished wound-healing capacity and decreased the migrated cells (Fig. 4), suggesting that PIK3R1 promotes migration by HCC cells in vitro.

\section{Downregulation of PIK3R1 expression increases cell apoptosis}

Additionally, flow cytometry was used to examine cell apoptosis. Compared with control group, the apoptotic rate of MHCC97H-si PIK3R1 and HCCLM3-si PIK3R1 cells were significantly increased (Fig. 5a, b). Thus, the down-regulation of PIK3R1 expression by siRNA increases apoptosis in HCCLM3 and MHCC97H cells.

\section{Knockdown of PIK3R1 downregulated p-PI3K, p-AKT,} and p-mTOR expressions in MHCC97H and HCCLM3 cells In order to investigate the possible mechanism of PIK3R1 in HCC, MHCC97H and HCCLM3 cells were transfected 
Table 1 Correlations between PIK3R1 expression and clinicopathologic characteristics in HCC cancer

\begin{tabular}{|c|c|c|c|c|}
\hline \multirow{2}{*}{$\begin{array}{l}\text { Clinicopathologic } \\
\text { Characteristics }\end{array}$} & \multirow{2}{*}{$\begin{array}{l}\text { No. } \\
\text { of patients }\end{array}$} & \multicolumn{2}{|l|}{ PIK3R1 } & \multirow[t]{2}{*}{$p$ value } \\
\hline & & High & Low & \\
\hline \multicolumn{5}{|l|}{ Age (year) } \\
\hline$>60$ & 63 & $34(54.0 \%)$ & $29(46.0 \%)$ & \multirow[t]{2}{*}{0.548} \\
\hline$\leq 60$ & 29 & $16(55.2 \%)$ & $13(44.8 \%)$ & \\
\hline \multicolumn{5}{|l|}{ Gender } \\
\hline Male & 57 & $26(45.6 \%)$ & $31(54.4 \%)$ & \multirow[t]{2}{*}{0.484} \\
\hline Female & 35 & $15(42.9 \%)$ & $20(57.1 \%)$ & \\
\hline \multicolumn{5}{|l|}{ Tumor size (cm) } \\
\hline$<3$ & 38 & $21(55.3 \%)$ & $17(44.7 \%)$ & \multirow[t]{2}{*}{0.503} \\
\hline$\geq 3$ & 54 & $31(57.4 \%)$ & $23(42.6 \%)$ & \\
\hline \multicolumn{5}{|l|}{ Lymphatic metastasis } \\
\hline NO & 59 & $24(40.7 \%)$ & $35(59.3 \%)$ & \multirow[t]{2}{*}{$0.029^{*}$} \\
\hline $\mathrm{N} 1-\mathrm{N} 4$ & 33 & $21(63.6 \%)$ & $12(36.4 \%)$ & \\
\hline \multicolumn{5}{|l|}{ Distal metastasis } \\
\hline MO & 79 & $21(26.6 \%)$ & $58(73.4 \%)$ & \multirow[t]{2}{*}{$0.004^{* *}$} \\
\hline M1 & 13 & $9(69.2 \%)$ & $4(30.8 \%)$ & \\
\hline \multicolumn{5}{|l|}{ TNM stage } \\
\hline $0 \& \mid \& \|$ & 64 & $19(29.7 \%)$ & $45(70.3 \%)$ & \multirow[t]{2}{*}{$0.002^{* *}$} \\
\hline III \& IV & 28 & $18(64.3 \%)$ & $10(35.7 \%)$ & \\
\hline
\end{tabular}

TNM stage pathologic tumor, node, metastasis stage ${ }^{*} p<0.05,{ }^{* *} p<0.01$ with PIK3R1 siRNAs, respectively. The results showed that the protein expression levels of $\mathrm{p}-\mathrm{PI} 3 \mathrm{~K}, \mathrm{p}-\mathrm{AKT}$, and p-mTOR were downregulated in si-PIK3R1 group compared with siRNA NC group. These data demonstrated that knockdown of PIK3R1 by siRNAs inhibited p-PI3K, $\mathrm{p}$-AKT, and p-mTOR expressions in $\mathrm{MHCC} 97 \mathrm{H}$ and HCCLM3 cells (Fig. 6).

\section{Discussion}

PIK3R1 has been shown to play important roles in many developmental processes, including cancer. In recent years, researchers found that PIK3R1 was abnormally expressed in various tumors is related to increased cell proliferation and invasion and reduced apoptosis [10]. Although PIK3R1 has been proved to function as an oncogene in many malignances, the relationship between PIK3R1 and HCC has not been fully elucidated. In the present study, we employed immunohistochemistry, qRT-PCT and western blot to accurately detect the expression level of PIK3R1 in HCC tissues. Besides, we used two $\mathrm{HCC}$ cell lines to explore the possible regulatory mechanism of PIK3R1 in the tumorigenesis of HCC. We described here that both the protein and mRNA levels of PIK3R1 was highly expressed in most human primary HCC tissues, whereas lowly expressed in adjacent

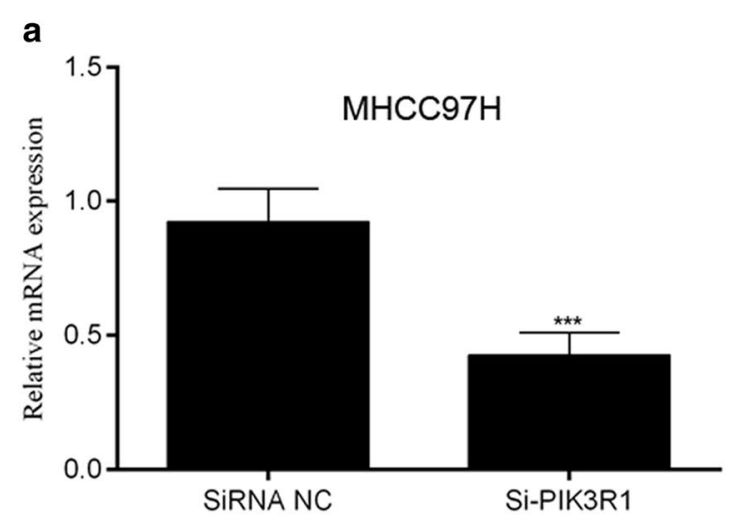

C

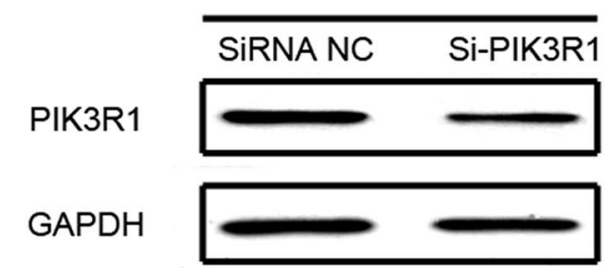

b

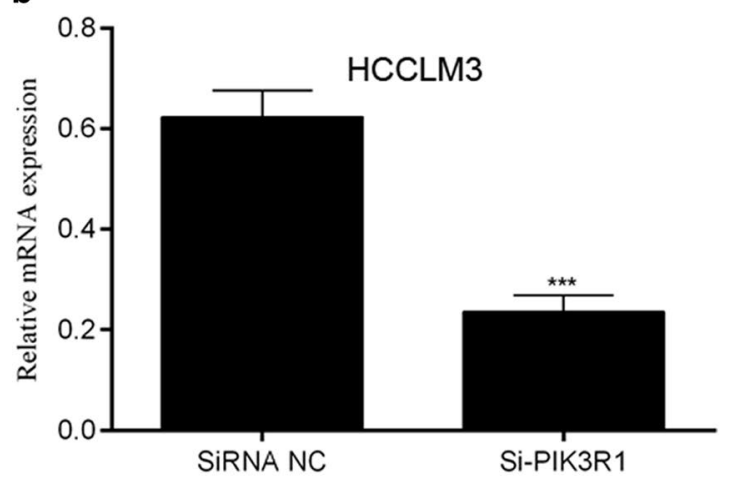

d

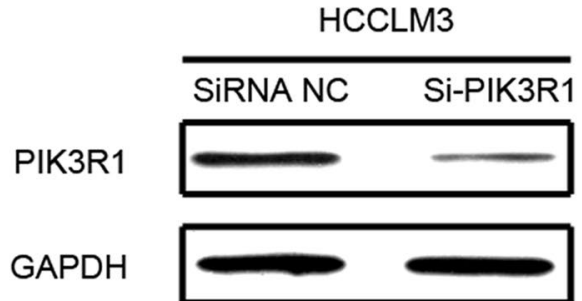

Fig. 2 MHCC97H and HCCLM3 cells were infected with PIK3R1 siRNA or siRNA NC. a, b PIK3R1 mRNA expression was analyzed by qRT-PCR; c, d MHCC97H and HCCLM3 cells were infected with PIK3R1 siRNA or siRNA NC. PIK3R1 protein expression was analyzed by western blotting. ${ }^{* * *} p<0.001$ 
a

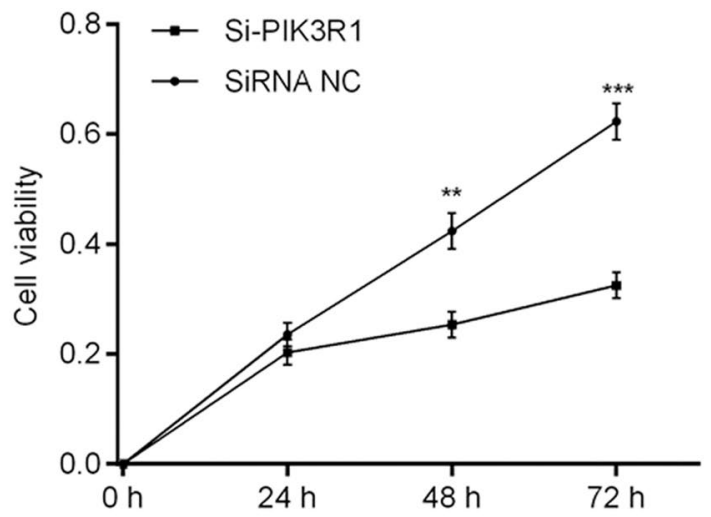

C
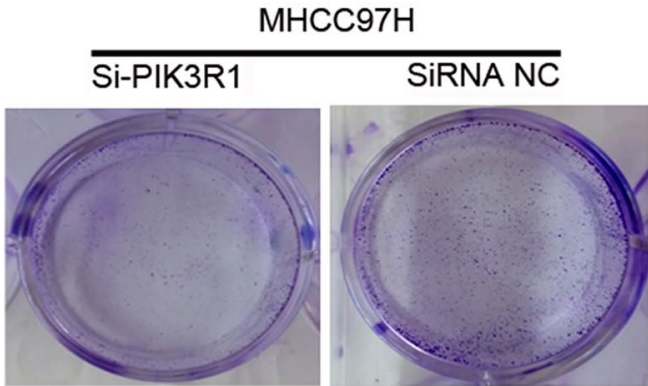

e

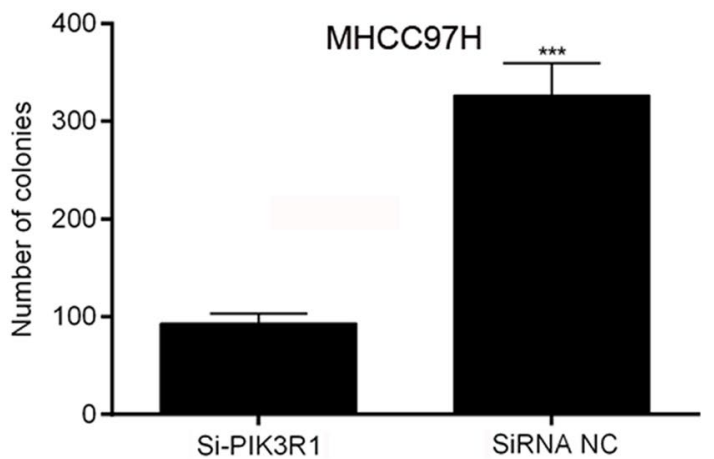

b

\section{HCCLM3}

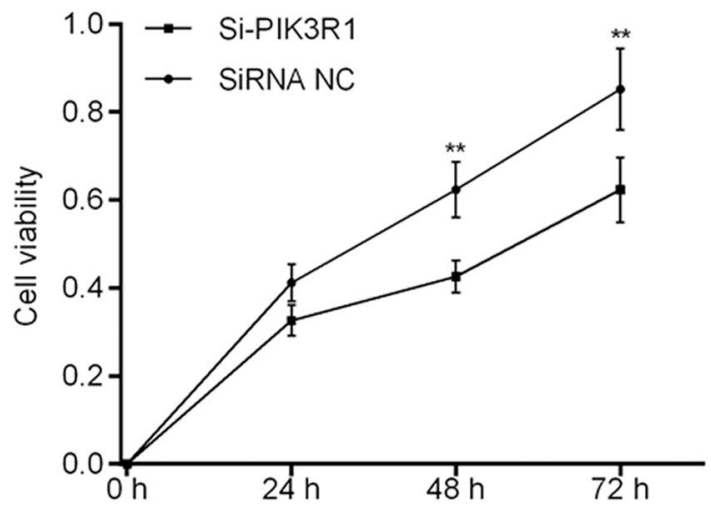

d

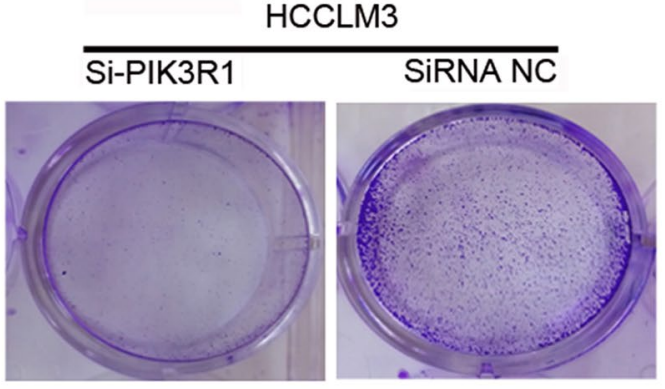

f

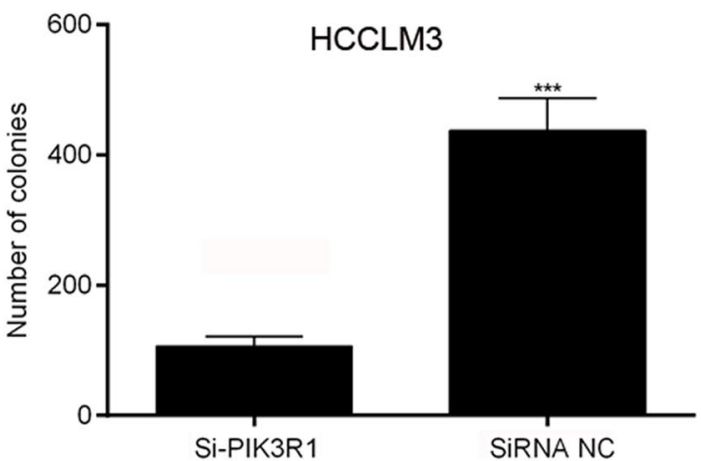

Fig. 3 PIK3R1 knockdown inhibits the proliferation of MHCC97H and HCCLM3 cells. a, b Cell proliferation was measured by MTT assay; c-e colony formation was analyzed by colony-formation assay and quantification of colonies number. ${ }^{* *} p<0.01,{ }^{* * *} p<0.001$

normal liver tissues, suggesting the important roles of PIK3R1 in human HCC tumorigenesis.

Cancer has biological characteristics including abnormal cell differentiation and proliferation, uncontrolled growth, infiltration and metastasis. The occurrence of cancer is a complex process with multiple factors and steps, which can be divided into three processes: carcinogenic, tumor promotion and progressive [11, 12]. In addition, apoptosis, serves as a crucial part of biological process, is a gene-controlled cell-independent death process, and a vital mechanism to maintain stable internal environment [13]. In our study, we proved that PIK3R1 expression was significantly increased in our six HCC cell lines, especially in HCCLM3 and MHCC97H cells, which exhibited the much higher metastatic ability, revealing that PIK3R1 may promote the tumor metastasis. Therefore, we selected HCCLM3 and MHCC97H cells to further investigate the involvement of PIK3R1 in HCC progression. We had carefully evaluated the direct effect of PIK3R1 on the ability of cell proliferation, apoptosis and migration. Inhibition of PIK3R1 was found to suppress the proliferation and colony forming capability 
a
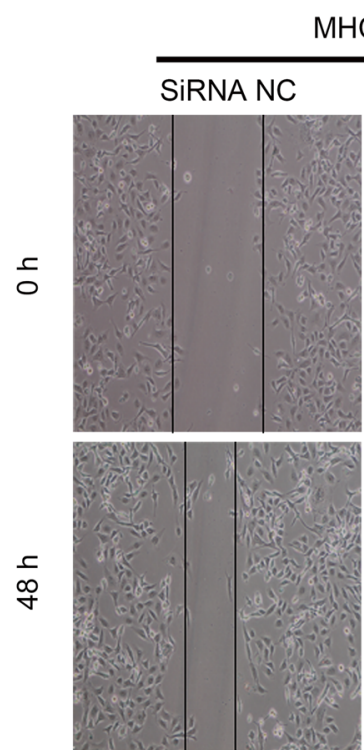

c
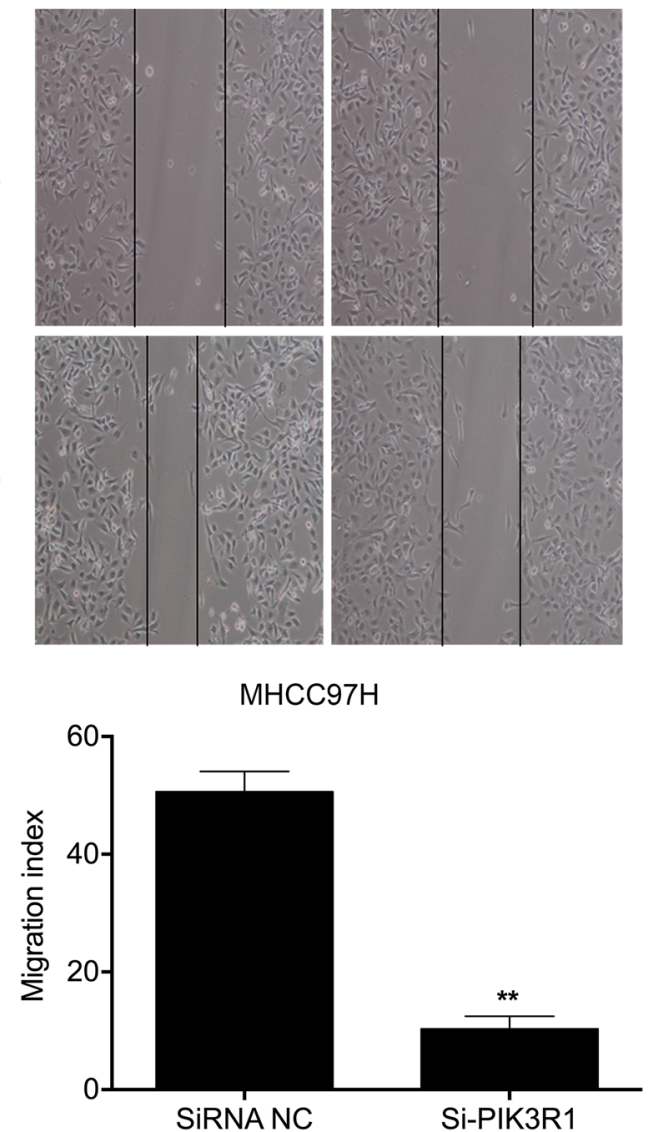

b
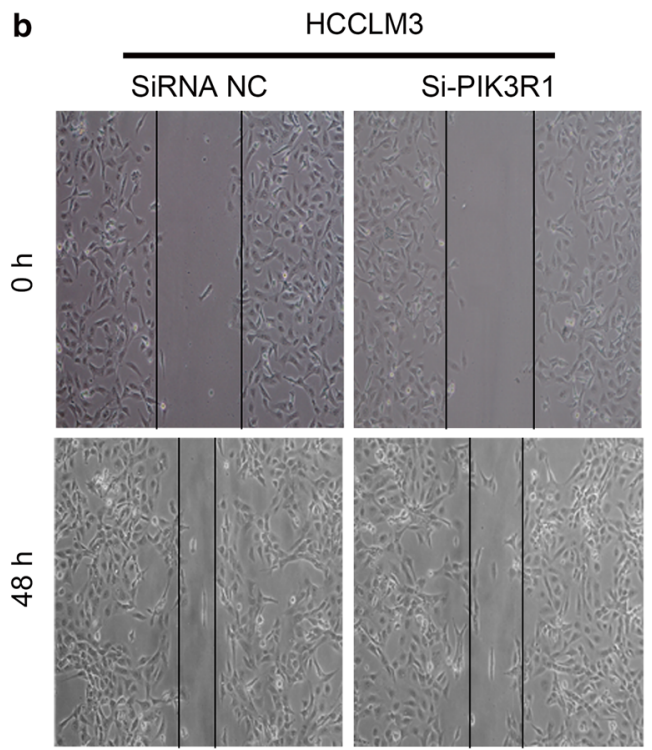

d

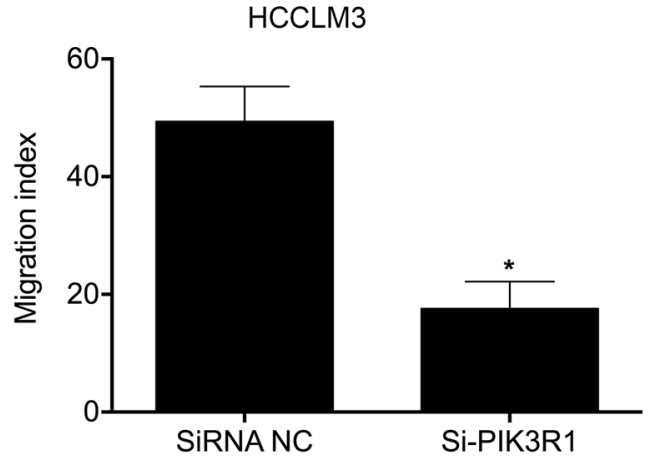

e $\mathrm{MHCC} 97 \mathrm{H}$
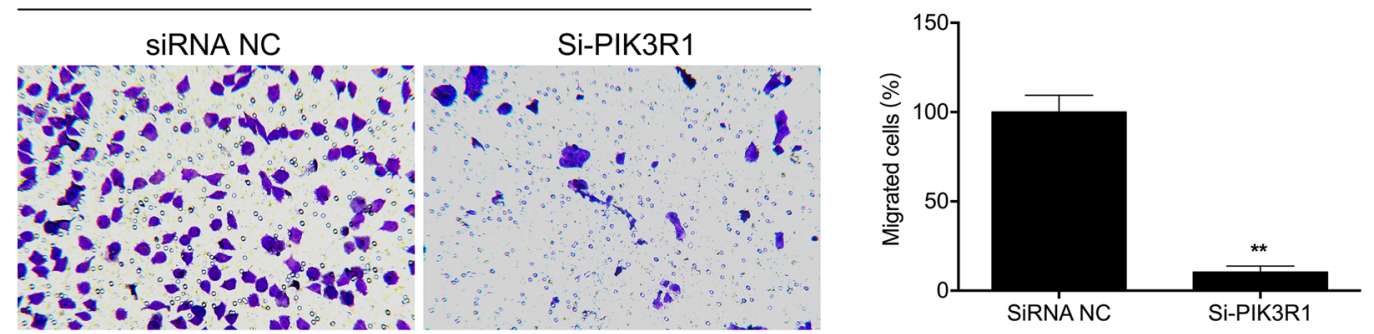

$\mathbf{f}$

HCCLM3
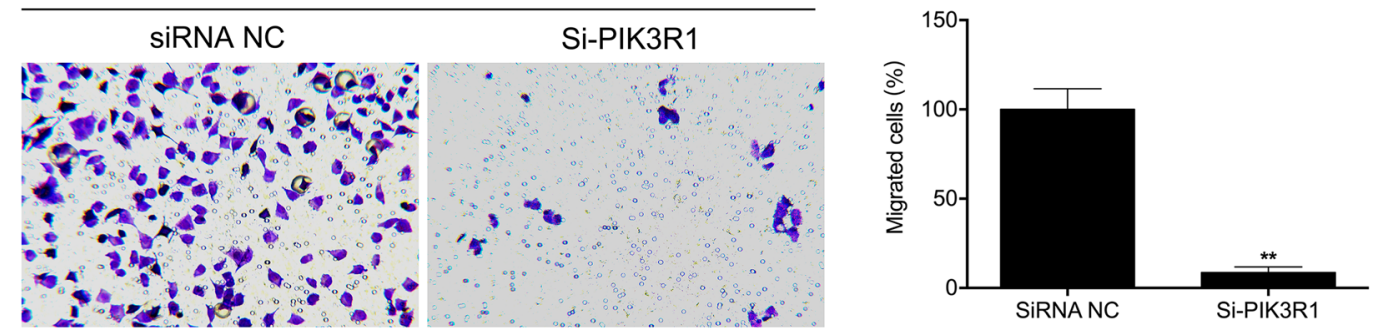

Fig. 4 PIK3R1 knockdown inhibits the migration and invasion of MHCC97H and HCCLM3 cells. $\mathbf{a}$, b Cell migration was measured by wound healing assays; $\mathbf{c}, \mathbf{d}$ quantification of migration index. e, $\mathbf{f}$ Cell migration ability was measured by transwell assay in MHCC97H and HCCLM3 cells after PIK3R1 knockdown. The migrated cells were calculated. ${ }^{* *} p<0.01$ vs. siRNA NC group 


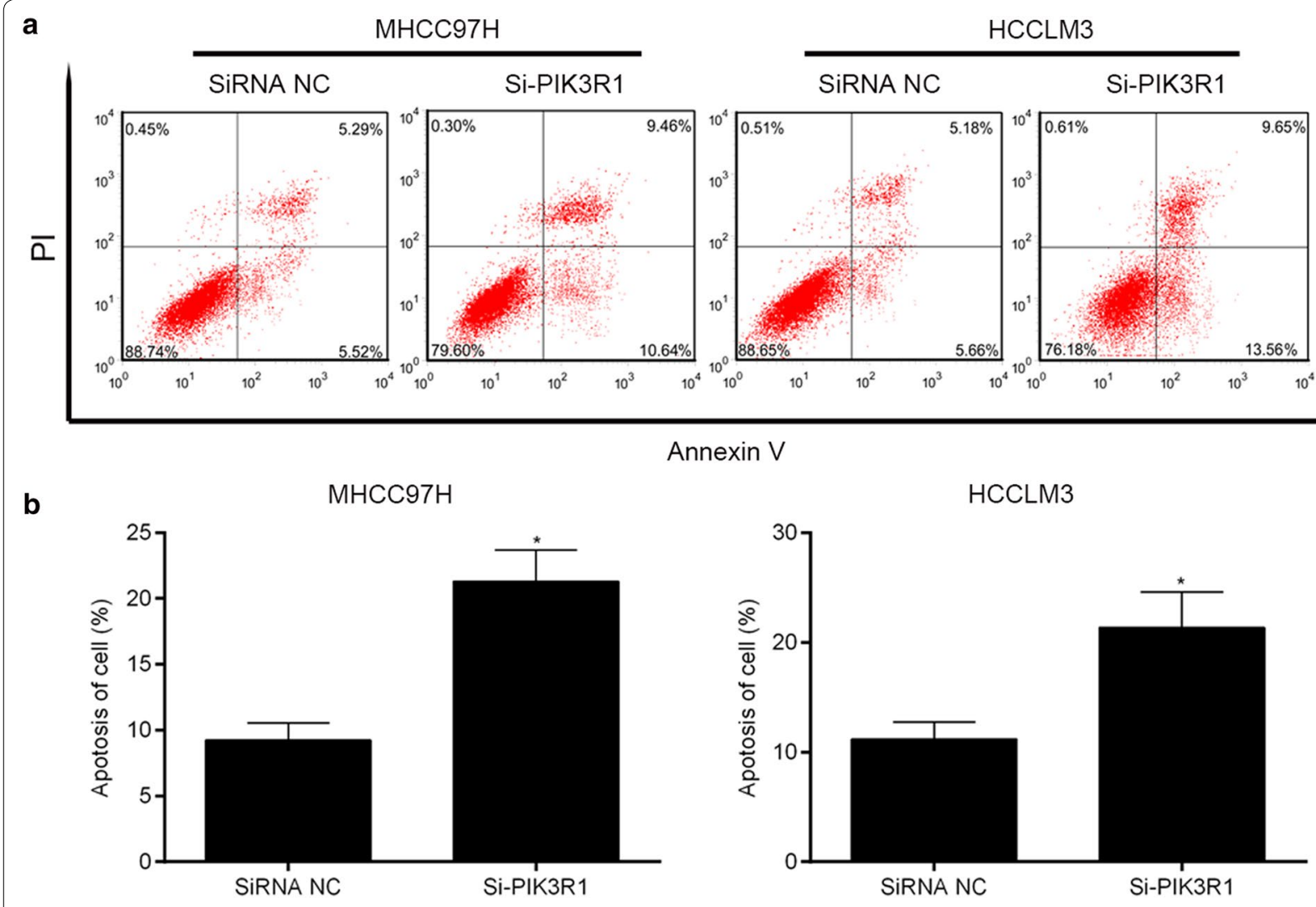

Fig. 5 Down-regulation of PIK3R1 expression by siRNA increases apoptosis in MHCC97H and HCCLM3 cells. a Flow cytometry detection of cell apoptosis; $\mathbf{b}$ quantification of apoptosis

of HCCLM3 and MHCC97H cells compared with control-siRNA cells. In addition, we demonstrated that knockdown of PIK3R1 inhibited the migration and promoted apoptosis of HCC cell lines. Combining with the previous reports, these observations further confirmed the oncogenic roles of PIK3R1 in HCC. The downregulation of PIK3R1 led to growth inhibition of HCC cells, which might be correlated with cell arrest in G2/M phase of cell cycle and apoptosis enhancement $[14,15]$. As we know, PI3K is a dimeric enzyme consisting of a catalytic (p110) and a regulatory subunit (p85 $\alpha$ ). p85 $\alpha$, encoded by PIK3R1, is reported to be an oncogene in ovarian, colorectal and prostate cancers, so exploring the role of $\mathrm{p} 85 \alpha$ in HCC may provide unique insights into activation of PI3K/AKT pathway $[16,17]$. Previous researches had revealed that PIK3R1 silencing could repress Huh7 proliferation, which is consistent with the other research that the deletion or reduction of PIK3R1 impaired B cell development and proliferation, delayed embryonic body development and inhibited cell adhesion [18, 19]. In addition, studies showed that PIK3R1 participated in the epithelial-mesenchymal transition of renal cancer cells [7]; PIK3R1 played an essential role kidney cancer [20];
PIK3R1 was involved in the migration and invasion of breast cancer byPI3K/AKT signaling [21]. Thus, PIK3R1 might be a potential target for cancer therapy.

A great deal of researches that showed that phosphatidylinositol 3-kinase (PI3K) signaling pathway is closely related to the occurrence and development of various human tumors, including HCC $[22,23]$. Protein kinase B (PKB/AKT), a serine/threonine (Ser/Thr) protein kinase, is the main effector of PI3K downstream [24]. The aberrant expression of AKT can be detected in multiple malignant tumor cells such as HCC [25-27]. The mechanistic target of rapamycin (mTOR), an atypical Ser/Thr protein kinase, is a downstream effector protein of AKT, which regulates transcription and protein synthesis, and has an important influence on growth and proliferation of tumor cells [28-30]. However, whether PIK3R1 can regulate the PI3K-AKT-mTOR signaling pathway in HCC cells has not been reported. In our study, we further demonstrated that knockdown of PIK3R1 obviously downregulated $\mathrm{p}$-PI3K, $\mathrm{p}$-AKT, and $\mathrm{p}$-mTOR expressions in HCC cells, suggesting that knockdown of PIK3R1 inhibited PI3K/AKT/mTOR pathway in HCC. 


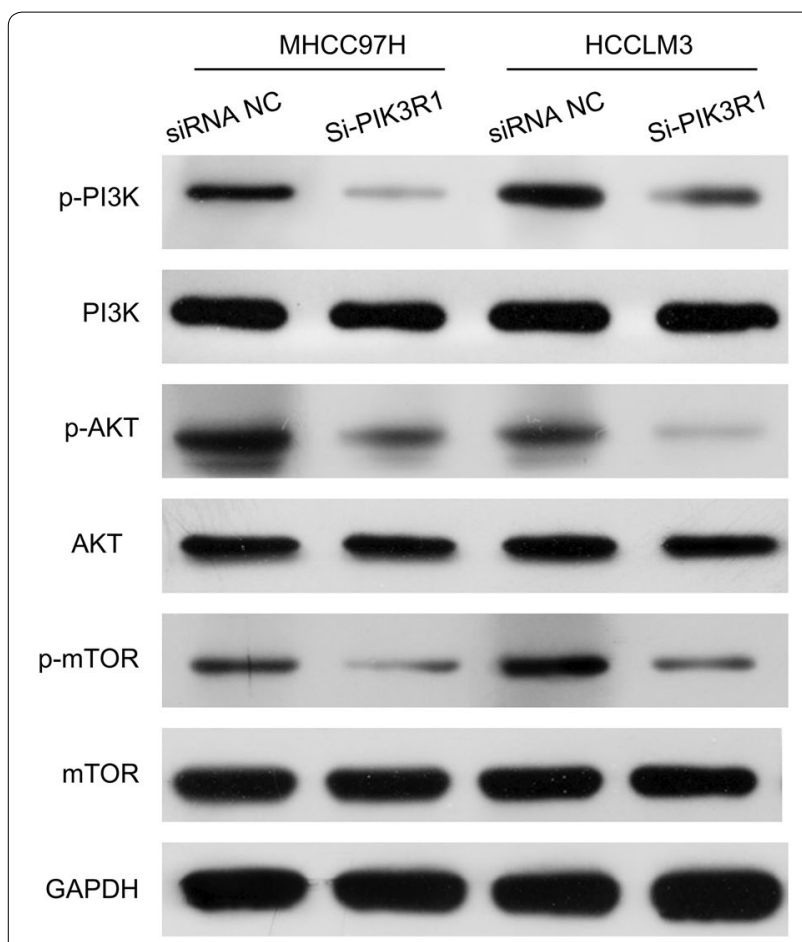

Fig. 6 Knockdown of PIK3R1 downregulated p-PI3K, p-AKT, and p-mTOR in MHCC97H and HCCLM3 cells. The protein expression levels of PI3K, p-PI3K, AKT, p-AKT, mTOR and p-mTOR were assessed by Western blot assay in MHCC97H and HCCLM3 cells after PIK3R1 knockdown. GAPDH was used as loading control

\section{Conclusions}

In conclusion, we found that PIK3R1 expression was upregulated in the majority of HCC clinical tissue specimens, silence of PIK3R1 suppressed cell proliferation, migration, and accelerated apoptosis of HCC cells. In addition, silence of PIK3R1 decreased p-PI3K, p-AKT, and $\mathrm{p}$-mTOR expressions in HCC. These findings provide information that will facilitate development of a novel therapeutic approach against HCC. However, further studies are needed to explore the possible mechanisms of PIK3R1 on HCC proliferation, apoptosis and migration. Moreover, it will be necessary to determine the deeper functions and mechanisms of PIK3R1 on HCC in vivo.

\section{Abbreviations}

PIK3R1: phosphoinositide-3-kinase, regulatory subunit 1; HCC: hepatocellular carcinoma; PI3K: phosphoinositide 3-kinase; EC: endometrial cancers; DAB: diaminobenzidine; SD: standard deviation.

\section{Authors' contributions}

All authors have contributed significantly. XA, LX, and ZH carried out the studies. XA and LX analyzed the data. SZ (Shi Zhou), SZ (Shuai Zhang), TZ and TJ supervised the study, XA and LX wrote the manuscript. All authors read and approved the final manuscript.

\section{Author details}

${ }^{1}$ The Key Laboratory of Medical Molecular Biology, Guizhou Medical University, Guiyang 550002, China. ${ }^{2}$ Department of Digestive, Guiyang First People's
Hospital, Guiyang 550001, China. ${ }^{3}$ Department of Interventional Radiology, Affiliated Hospital of Guizhou Medical University, Guiyang 550002, China.

\section{Competing interests}

The authors declare that they have no competing interests.

\section{Availability of data and materials}

Please contact author for data requests.

\section{Consent for publication}

Not applicable.

\section{Ethics approval and consent to participate}

This study was approved by the ethics committee at the Guizhou Medical University.

\section{Funding}

This study was supported by the National Natural Science Foundation of China (No: 81560297) and the Scientific Cooperation in Guizhou Province (No: LH2016[7405]).

\section{Publisher's Note}

Springer Nature remains neutral with regard to jurisdictional claims in published maps and institutional affiliations.

Received: 9 August 2018 Accepted: 20 November 2018

Published online: 29 November 2018

\section{References}

1. Yang JD, Roberts LR. Hepatocellular carcinoma: a global view. Nat Rev Gastroenterol Hepatol. 2010;7(8):448.

2. El-Serag HB, et al. Diagnosis and treatment of hepatocellular carcinoma. Gastroenterology. 2008;134(6):1752-63.

3. Halleen JM, et al. Serum tartrate-resistant acid phosphatase 5b is a specific and sensitive marker of bone resorption. Clin Chem. 2001;47(3):597-600.

4. Ueki $\mathrm{K}$, et al. Positive and negative regulation of phosphoinositide 3-kinase-dependent signaling pathways by three different gene products of the p85alpha regulatory subunit. Mol Cell Biol. 2000;20(21):8035-46.

5. Taniguchi CM, Emanuelli B, Kahn CR. Critical nodes in signalling pathways: insights into insulin action. Nat Rev Mol Cell Biol. 2006;7(2):85-96.

6. Fruman DA, et al. Hypoglycaemia, liver necrosis and perinatal death in mice lacking all isoforms of phosphoinositide 3-kinase p85 alpha. Nat Genet. 2000;26(3):379-82.

7. Lin Y, et al. PIK3R1 negatively regulates the epithelial-mesenchymal transition and stem-like phenotype of renal cancer cells through the AKT/ GSK3beta/CTNNB1 signaling pathway. Sci Rep. 2015;5:8997.

8. Samuels $Y$, et al. High frequency of mutations of the PIK3CA gene in human cancers. Science. 2004;304(5670):554.

9. Chikara S, et al. Enterolactone alters FAK-Src signaling and suppresses migration and invasion of lung cancer cell lines. BMC Complement Altern Med. 2017;17(1):30.

10. Andrade VP, et al. Gene expression profiling of lobular carcinoma in situ reveals candidate precursor genes for invasion. Mol Oncol. 2015;9(4):772-82.

11. Jin $H, W u Y, \operatorname{Tan} X$. The role of pancreatic cancer-derived exosomes in cancer progress and their potential application as biomarkers. Clin Transl Oncol. 2017;19(8):921-30.

12. Mantia-Smaldone GM, Chu CS. A review of dendritic cell therapy for cancer: progress and challenges. BioDrugs. 2013;27(5):453-68.

13. Hassan M, et al. Apoptosis and molecular targeting therapy in cancer. Biomed Res Int. 2014;2014:150845.

14. Zhuo ZJ, et al. Novel betulin derivative induces anti-proliferative activity by G2/M phase cell cycle arrest and apoptosis in Huh7 cells. Oncol Lett. 2018;15(2):2097-104.

15. Chen $\mathrm{S}$, et al. miR-502 inhibits cell proliferation and tumor growth in hepatocellular carcinoma through suppressing phosphoinositide 
3-kinase catalytic subunit gamma. Biochem Biophys Res Commun. 2015;464(2):500-5.

16. Philp AJ, et al. The phosphatidylinositol 3 '-kinase p85a gene is an oncogene in human ovarian and colon tumors. Can Res. 2001;61(20):7426-9.

17. Zhu Q, et al. Phosphoinositide $3-\mathrm{OH}$ kinase $\mathrm{p} 85 \mathrm{a}$ and $\mathrm{p} 110 \beta$ are essential for androgen receptor transactivation and tumor progression in prostate cancers. Oncogene. 2008;27(33):4569.

18. Zheng Y, et al. miR-376a suppresses proliferation and induces apoptosis in hepatocellular carcinoma. FEBS Lett. 2012;586(16):2396-403.

19. LiT, et al. Comparison of gene expression in hepatocellular carcinoma, liver development, and liver regeneration. Mol Genet Genomics. 2010;283(5):485-92.

20. Wang YD, Sun ZL. Effects of miR-455 on PIK3R1 gene expression regulation and kidney cancer cell functions. Eur Rev Med Pharmacol Sci. 2017;21(15):3370-6.

21. Yan LX, et al. PIK3R1 targeting by miR-21 suppresses tumor cell migration and invasion by reducing PI3K/AKT signaling and reversing EMT, and predicts clinical outcome of breast cancer. Int J Oncol. 2016;48(2):471-84.

22. Fruman DA, et al. The PI3K pathway in human disease. Cell. 2017:170(4):605-35
23. Fruman DA, Rommel C. PI3K and cancer: lessons, challenges and opportunities. Nat Rev Drug Discov. 2014;13(2):140-56.

24. Lien EC, Dibble CC, Toker A. PI3K signaling in cancer: beyond AKT. Curr Opin Cell Biol. 2017;45:62-71.

25. Clark AR, Toker A. Signalling specificity in the Akt pathway in breast cancer. Biochem Soc Trans. 2014;42(5):1349-55.

26. Gdowski A, et al. Emerging therapeutics for targeting Akt in cancer. Front Biosci (Landmark Ed). 2016;21:757-68.

27. Mundi PS, et al. AKT in cancer: new molecular insights and advances in drug development. Br J Clin Pharmacol. 2016;82(4):943-56.

28. Guerrero-Zotano A, Mayer IA, Arteaga CL. PI3 K/AKT/mTOR: role in breast cancer progression, drug resistance, and treatment. Cancer Metastasis Rev. 2016;35(4):515-24.

29. Lien EC, Lyssiotis CA, Cantley LC. Metabolic reprogramming by the PI3KAkt-mTOR pathway in cancer. Recent Results Cancer Res. 2016;207:39-72.

30. Polivka J Jr, Janku F. Molecular targets for cancer therapy in the PI3K/AKT/ mTOR pathway. Pharmacol Ther. 2014;142(2):164-75.
Ready to submit your research? Choose BMC and benefit from:

- fast, convenient online submission

- thorough peer review by experienced researchers in your field

- rapid publication on acceptance

- support for research data, including large and complex data types

- gold Open Access which fosters wider collaboration and increased citations

- maximum visibility for your research: over $100 \mathrm{M}$ website views per year

At BMC, research is always in progress.

Learn more biomedcentral.com/submissions 